\title{
Surgical debranching for endovascular arch penetrating ulcer management
}

\author{
Tim Smith, Emma van der Weijde, Robin H. Heijmen \\ Department of Cardiothoracic Surgery, St. Antonius Hospital, Nieuwegein, The Netherlands \\ Correspondence to: Robin H. Heijmen, MD PhD. Department of Cardiothoracic Surgery, St. Antonius Hospital, Koekoekslaan 1, 3234 CM \\ Nieuwegein, The Netherlands. Email: r.heijmen@antoniusziekenhuis.nl.
}

Submitted Mar 08, 2019. Accepted for publication May 31, 2019.

doi: 10.21037/acs.2019.06.01

View this article at: http://dx.doi.org/10.21037/acs.2019.06.01

\section{Clinical vignette}

A 72-year-old male was referred to our unit because of a penetrating atherosclerotic ulcer (PAU) of the aortic arch with signs of contained rupture on CT. His relevant medical history comprised prior coronary bypass surgery 24 years earlier, and a recent episode of fever possibly due to local aortitis of the aortic arch, which was treated conservatively with antibiotics in the referring hospital. Recently, hoarseness was noted due to paralysis of the left recurrent laryngeal nerve. The patient was hemodynamically stable at time of admission to our unit.

Computed tomography showed a large PAU located in the inner curvature of the aortic arch with signs of contained rupture. The anatomy of the arch vessels consisted of a large bovine trunk giving rise to the brachiocephalic trunk (BCT) and the left common carotid artery (LCCA), with circumferential calcifications at the origin of the bovine trunk. The left subclavian artery was proximally occluded. Coronary angiography showed occluded vein grafts [to left anterior descending (LAD) and circumflex (CX)], and retrograde perfusion of the LAD. Echocardiography showed normal biventricular function and normal valvular function. The diameter of the iliacofemoral vessels appeared just large enough to allow passage of a 24 French device needed for the thoracic endovascular aortic repair (TEVAR) procedure. We routinely perform a transcranial color-coded duplex study to assess the functional anatomy of the Circle of Willis, which in this patient appeared to be complete (1).

\section{Surgical techniques}

\section{Preparation}

We planned to perform a complete aortic arch debranching to facilitate TEVAR with the stentgraft covering the total arch and thus, excluding the PAU.

To minimize the risk of iatrogenic dissection of the ascending aorta due to partial clamping, and to guarantee adequate cerebral perfusion throughout the debranching procedure, we planned to perform the procedure with assistance of cardiopulmonary bypass (CPB), with the right subclavian artery (RSA) used for arterial inflow. This facilitates both safe performance of the proximal anastomosis (ascending aorta to the main trunk of the bifurcation graft) using a large side-biting clamp while the heart is (partially) unloaded, and additionally, a relatively low pressure, reduced pulsatility flow in the ascending aorta during the procedure. Further, if the RSA is used for arterial inflow of the $\mathrm{CPB}$ circuit, it is possible to deliver unilateral antegrade selective cerebral perfusion (ASCP) during the anastomosis between the bifurcation graft and the distal stump of the BCT, by clamping the origin of the $\mathrm{BCT}$ and adjusting arterial flow rate.

Since no adequately sized off-the-shelf bifurcation prosthesis was available, we decided to hand-sew a bifurcation graft consisting of a $12 \mathrm{~mm}$ Dacron vascular prosthesis as the main body and branch to supply the BCT, and an $8 \mathrm{~mm}$ branch to supply the LCCA. Because we were uncertain about the possibility of delivering the stentgraft in 


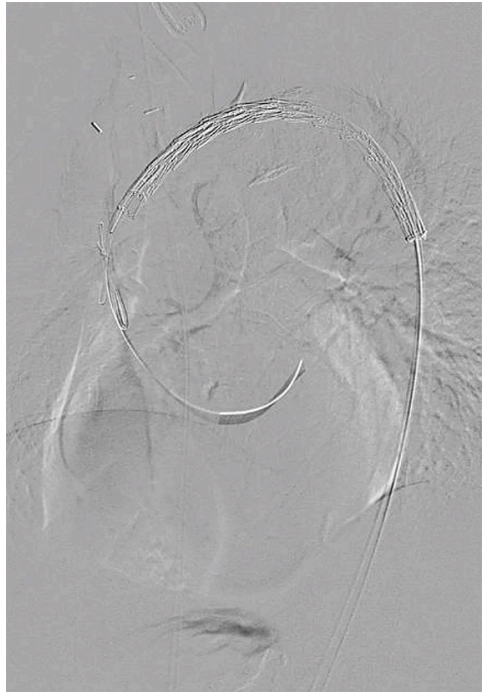

Figure 1 Insertion of a TEVAR stentgraft under fluoroscopic guidance. TEVAR, thoracic endovascular aortic repair.

a retrograde manner via the femoral artery (because of the marginal diameter), we anastomosed a $10 \mathrm{~mm}$ side branch proximal to the main body to eventually be able to safely deliver the stentgraft in antegrade fashion through this branch, if necessary.

\section{Exposition}

The procedure was started with surgical exposure and cannulation of the RSA. We prefer to anastomose an $8 \mathrm{~mm}$ Dacron vascular graft to the RSA for arterial inflow instead of direct cannulation to minimize the risk of vascular injury. Before sternal re-entry, a guidewire was placed via the femoral vein into the superior caval vein to aid venous cannulation in case of unexpected severe bleeding at resternotomy. After safe sternal reentry and adhesiolysis, a staged venous cannula was placed via the right femoral vein into the superior vena cava over the guidewire under transoesophageal echocardiography guidance, and CPB was instituted.

\section{Operation}

The debranching was started with the proximal anastomosis (i.e., between the ascending aorta and the main trunk of the bifurcation graft) using a large side-biting clamp. The heart was unloaded and the systemic pressure was kept around $60 \mathrm{mmHg}$. After careful completion of the anastomosis and de-airing the graft, the side-biting clamp was released and flow of $\mathrm{CPB}$ was reduced to approximately 1 liter for right-sided ASCP via the BCT (at normothermia). At the same time, the origin of the BCT was clamped with two vascular clamps and the vessel was transected in between. Adequate cerebral flow in the left and right middle cerebral artery was confirmed with transcranial duplex, and cerebral oximetry. Next, the end-to-end anastomosis between the $12 \mathrm{~mm}$ arm of the bifurcation graft and the origin of the BCT was performed. After de-airing, antegrade flow via the prosthesis was restarted and ASCP was discontinued. The stump of the BCT was ligated. The debranching was completed with the anastomosis between the $8 \mathrm{~mm}$ arm of the prosthesis and the origin of the LCCA, which was performed during temporary clamping of the LCCA. Transcranial duplex confirmed that adequate collateral flow via the intact Circle of Willis was maintained in the left middle cerebral artery during this phase. Finally, we ligated the stump of the LCCA and decannulated the RSA. The proximal anastomosis was marked with a radiopaque marker to aid placement of the TEVAR.

Next, safe introduction of a TEVAR stentgraft (Conformable GORE ${ }^{\circledR}$ TAG $^{\circledR} 40 \mathrm{~mm} \times 150 \mathrm{~mm}$ ) in retrograde fashion via the right femoral artery was performed under fluoroscopic guidance (Figure 1). The complete arch was covered, respecting the radiopaque marker that we placed at the proximal anastomosis. Completion angiography confirmed adequate position of the graft and sealing of the PAU, with uncompromised flow via the bifurcation graft (Figure 2).

\section{Completion}

After reversal of heparin, conventional closure of the sternum and the femoral and subclavian access sites was performed.

\section{Comments}

\section{Clinical results}

The post-operative course was uneventful. The patient was transferred to the ward on the fifth post-operative day and discharged home on the 11th post-operative day. The first post-operative CT-scan showed a satisfactory result (Video).

\section{Advantages and caveats}

Penetrating ulcers are most frequently seen in the mid and 


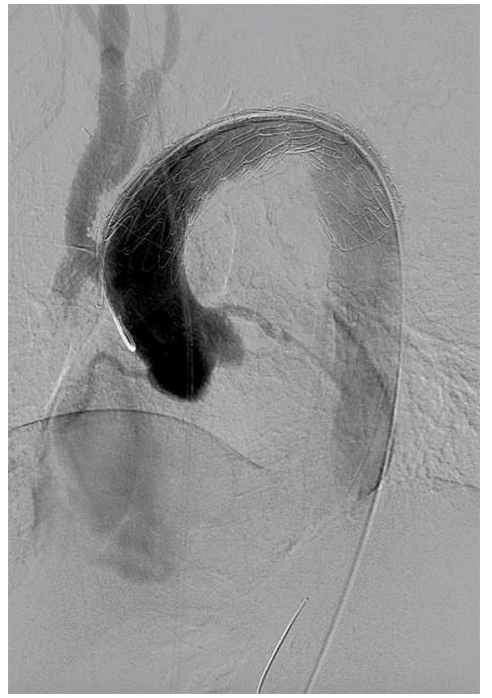

Figure 2 Completion angiography confirming adequate position of the graft and sealing of the PAU. PAU, penetrating atherosclerotic ulcer.

lower descending thoracic aorta, where endovascular repair is relatively simple because of the absence of important branching arteries besides intercostal arteries feeding the spinal cord (2). Penetrating ulcers in the aortic arch are rare-only $7 \%$ of all described PAU's are located in the arch-and complexity of treatment is due to the localization of the disease (3).

If TEVAR can be performed with adequate sealing in zone 2 [i.e., between the LCCA and left subclavian artery (LSA)], the LSA may be covered in case of adequate collateral perfusion. Nonetheless, we have a low threshold in revascularizing the LSA, certainly in case of a left internal mammary artery bypass graft, absent or hypoplastic right vertebral artery, or left vertebral artery (LVA) terminating in a posterior inferior cerebellar artery. The latter anatomical variant of the LVA may also complicate proper proximal seating of the stentgraft.

If sealing in zone 1 (i.e., between the BCT and LCCA) is necessary, remaining options are surgical debranching, extra-anatomical bypasses (3), or scalloped or fenestrated percutaneous devices.

Sealing in zone 0 (ascending aorta), as was necessary in the present case due to a short proximal landing zone, confronts the clinician with other technical challenges $(4,5)$. When choosing a debranching strategy with the proximal anastomosis in the ascending aorta, several options remain; the first is to perform the proximal anastomosis using a side-biting clamp, with its inherent risk of iatrogenic type-A dissection, which may be reduced by performing this anastomosis in an (partially) unloaded beating heart situation as described. A more invasive but elegant alternative is to perform a supracoronary ascending aorta replacement on the cardioplegic heart, to serve as a safe take-off zone for the debranching prosthesis. In this construction it is advisable to perform the proximal anastomosis as proximal (i.e., close to the sinotubular junction) as possible in order to preserve enough length for sealing, especially if the PAU is located in the inner curvature of the arch.

Probably the most invasive option is to perform a complete arch replacement with—if necessary—a Frozen Elephant Trunk procedure, necessitating both cardioplegic and circulatory arrest, but with the advantage of complete resection of all pathological arch tissue. Because of the age and comorbid conditions of our patient, we opted for endovascular repair after surgical debranching, preventing the need for both cardiac and systemic circulatory arrest.

\section{Acknowledgments}

None.

\section{Footnote}

Conflicts of Interest: The authors have no conflicts of interest to declare.

\section{References}

1. Smith T, Jafrancesco G, Surace G, et al. A functional assessment of the circle of Willis before aortic arch surgery using transcranial Doppler. J Thorac Cardiovasc Surg 2019. [Epub ahead of print].

2. Erbel R, Aboyans V, Boileau C, et al. 2014 ESC Guidelines on the diagnosis and treatment of aortic diseases: Document covering acute and chronic aortic diseases of the thoracic and abdominal aorta of the adult. The Task Force for the Diagnosis and Treatment of Aortic Diseases of the European Society of Cardiology (ESC). Eur Heart J 2014;35:2873-926.

3. Nathan DP, Boonn W, Lai E, et al. Presentation, complications, and natural history of penetrating atherosclerotic ulcer disease. J Vasc Surg 2012;55:10-5.

4. D'Annoville T, Ozdemir BA, Alric P, et al. Thoracic Endovascular Aortic Repair for Penetrating Aortic Ulcer: 
Literature Review. Ann Thorac Surg 2016;101:2272-8.

5. Canaud L, Hireche K, D'annoville T, et al. Hybrid aortic arch repair for a ruptured and infected penetrating

Cite this article as: Smith T, van der Weijde E, Heijmen RH. Surgical debranching for endovascular arch penetrating ulcer management. Ann Cardiothorac Surg 2019;8(4):512-515. doi: 10.21037/acs.2019.06.01 atherosclerotic ulcer of the aortic arch. Ann Vasc Surg 2011;25:266.e5-7. 